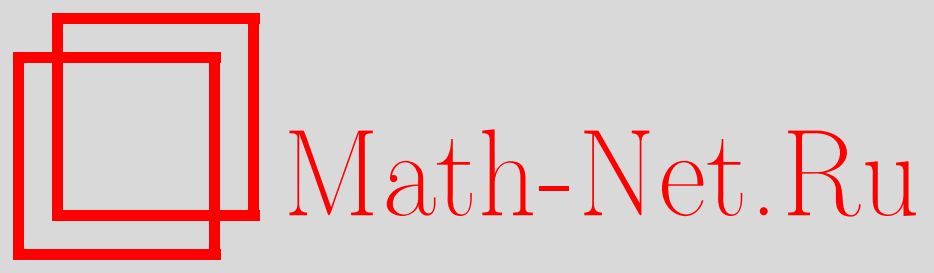

Е. А. Давыдов, Полиномиальные интегралы движения в теориях дилатонной гравитации, ТМФ, 2015, том 183, номер 1, 138-151

DOI: https://doi.org/10.4213/tmf8774

Использование Общероссийского математического портала Math-Net.Ru подразумевает, что вы прочитали и согласны с пользовательским соглашением http://www.mathnet.ru/rus/agreement

Параметры загрузки:

IP : 52.6 .47 .48

26 апреля 2023 г., 18:30:43

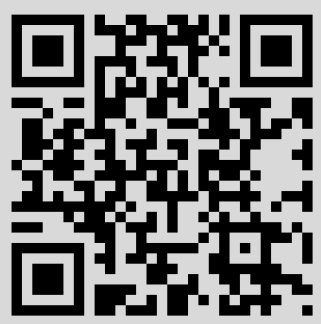




\section{ПОЛИНОМИАЛЬНЫЕ ИНТЕГРАЛЫ ДВИЖЕНИЯ В ТЕОРИЯХ ДИЛАТОННОЙ ГРАВИТАЦИИ}

Исследование современных моделей в гравитации и космологии содержит этап анализа сопутствующих нелинейных динамических систем. Как правило, такие системы не являются интегрируемыми, однако зачастую они допускают наличие дополнительных интегралов движения. На основе характерных свойств динамических систем, возникающих в теориях дилатонной гравитации, сформулирован универсальный алгоритм поиска полиномиальных по импульсу интегралов движения. С его помощью теории дилатонной гравитации с одним скалярным полем исследованы на наличие линейных и квадратичных интегралов движения.

Ключевые слова: гравитация, динамические системы, интегрируемость, полиномиальные интегралы.

DOI: $10.4213 / \operatorname{tmf} 8774$

\section{1. ВВЕДЕНИЕ}

Современные астрофизические данные [1], [2] свидетельствуют о том, что Вселенная эволюционирует достаточно нетривиальным образом. Простейших механизмов, таких как инфляция с единственным скалярным полем (инфлатоном) и темная энергия с космологической постоянной, вероятно, недостаточно для описания наблюдаемой динамики [3]-[6]. Одновременно успехи в разработке новых моделей в супергравитации, теории струн и многих других теориях, обобщающих теорию гравитации Эйнштейна за счет введения новых полей [7]-[10], других вариантов действия [11], [12] или геометрии пространства-времени [13], [14], приводят к пониманию того, что сложные нелинейные и нетривиальные конструкции не являются экзотикой, а могут иметь практическое применение для описания реальной Вселенной.

Для работы с такими разнообразными и сложными моделями достаточно хорошо подходят методы дилатонной гравитации, для краткости мы будем называть так обобщенные дилатонные теории гравитации с дополнительными полями материи в $1+1$ измерениях [15], [16]. В основе дилатонной гравитации лежит размерная

* Объединенный институт ядерных исследований, Дубна, Московская обл., Россия. E-mail: davydov@theor.jinr.ru 
редукция реальной многомерной теории к $1+1,1+0$ или $0+1$ измерениям с учетом пространственных симметрий. В результате получается эффективная теория, в которой на равных фигурируют простейшая двумерная метрика и поля, имеющие как материальную, так и геометрическую природу. Благодаря этому оказывается возможным применить традиционные методы лагранжева или гамильтонова формализма для исследования пространства решений. Построив классические решения, можно затем провести их квантование, что, как правило, невозможно сделать напрямую для оригинальной многомерной теории гравитации.

Полезно также изучать дилатонную гравитацию саму по себе, назависимо от порождающих ее "полноценных" теорий гравитации. Действительно, сравнение с наблюдательными данными фактически происходит именно на уровне этой эффективной теории, на пространстве ее решений. Поэтому можно, не ограничивая себя рамками какой-то конкретной теории гравитации, выяснить, какие модели дилатонной гравитации в принципе подходят для решения вопросов, возникающих в современной космологии. А затем уже можно устанавливать связь между найденными эффективными моделями и реальными фундаментальными теориями гравитации.

Следуя этому подходу, мы изучаем вопрос о существовании первых интегралов движения в достаточно широком классе моделей дилатонной гравитации. Действительно, знание первых интегралов позволяет выделять интегрируемые и частично интегрируемые модели, упрощает анализ пространства решений и указывает на скрытые симметрии.

Можно заметить, что классические методы исследования динамических систем зачастую применяются к моделям в гравитации в общем виде, без учета их характерных отличительных особенностей. Во-первых, для теорий гравитации характерно большое разнообразие эффективных моделей, существенно разных как по построению, так и по видам размерных редукций многомерных теорий к эффективным динамическим системам. Формализм общей теории относительности допускает и даже поощряет выбор различных калибровок, так что динамические системы для одной и той же модели выглядят по-разному в зависимости от выбора калибровки и динамических переменных [17]. Во-вторых, при гамильтоновой формулировке теории гравитации в подходе Арновитта-Дезера-Мизнера возникают динамические системы со связями, которые необходимо аккуратно учитывать.

Таким образом, представляется естественным при исследовании динамических систем в дилатонной гравитации подобрать инструментарий, который был бы независим от выбора переменных и калибровок. В частности, алгоритмы поиска интегралов движения, как правило, требуют фиксации определенного класса моделей, например движение частиц или динамика осцилляторов [18]-[21], определенные специфические системы типа системы Драша [22], а также многие другие [23], [24]. Однако в дилатонной гравитации такие естественные изменения, как выбор другой калибровки или другой размерной редукции, заметно меняют структуру лагранжиана. Нам будет удобно сформулировать алгоритм поиска интегралов движения в инвариантном виде, не зависящем от подобных преобразований.

Мы воспользуемся алгоритмом Уиттекера [18], который основывается на том, что гамильтонианы физических моделей, как правило, полиномиальны по импульсам. Соответственно, скобка Пуассона полиномиального гамильтониана и полиномиального же (по импульсам) выражения также даст полином по импульсам. Если 
приравнять нулю коэффициенты последнего полинома, то получатся условия сохранения первого интеграла.

Свойство полиномиальности по импульсам выполняется и в дилатонной гравитации достаточно общего вида. Однако в гравитации вследствие координатной инвариантности в гамильтоновой формулировке возникают дополнительные связи. В частности, для динамических систем гамильтониан должен быть равен нулю на уравнениях движения. Поэтому можно расширить алгоритм Уиттекера и искать такие полиномы, что их скобка Пуассона с гамильтонианом равнялась бы не полиному с нулевыми коэффициентами, а была пропорциональна гамильтониану. Тогда на уравнениях движения выполнение гамильтоновой связи повлечет также сохранение исходного полинома как первого интеграла.

Поэтому в разделе 2 мы применим известный математический формализм симметрической алгебры для построения на основе алгоритма Уиттекера модифицированного алгоритма, который будет записан в инвариантном виде и позволит учесть гамильтонову связь. Затем в разделе 3 с его помощью мы будем исследовать модели дилатонной гравитации с одним скалярным полем на наличие линейных и квадратичных интегралов движения. Такой класс моделей дилатонной гравитации может служить, например, для описания аффинного обобщения теории Эйнштейна [25], космологии со скалярным или векторным полем [14], решений типа черных дыр и гравитационных волн [26]-[28] и т.д. Знание линейного интеграла движения позволяет выделить циклическую координату и редуцировать конфигурационное пространство, что упрощает анализ модели. Наличие же дополнительных квадратичных интегралов соответствует нетривиальным симметриям, которые бывает непросто найти из каких-либо общих соображений. Однако это составляет важный шаг на пути к построению реалистичных интегрируемых моделей, что является достаточно редким случаем в моделях гравитации.

\section{2. ПОЛИНОМИАЛЬНЫЕ ИНТЕГРАЛЫ ДВИЖЕНИЯ В КОНСЕРВАТИВНЫХ СИСТЕМАХ}

Как мы отмечали выше, динамика в большинстве физических моделей описывается гамильтонианом, полиномиальным по импульсам. И в таком случае наиболее естественно искать первые интегралы в виде выражений, также полиномиальных по импульсам [18], [23], [24]. В настоящем разделе мы сформулируем алгоритм такого поиска в виде, подходящем для исследования достаточно общего класса динамических систем, вытекающих из современной теории гравитации и космологии. Мы будем учитывать два существенных свойства гравитационных моделей: наличие гамильтоновых связей и значительную свободу в выборе конкретного представления для соответствующей динамической системы. Заметим, что такой подход может быть удобен и для моделей, возникающих в других областях физики, где могут возникать другие характерные виды связей и наблюдается большой выбор используемых калибровок.

Для этих целей мы воспользуемся известными математическими конструкциями, которые, однако, применяются не столь часто. Для гамильтоновой системы будем 
обозначать векторы координат фазового пространства как $q=\left(q^{1}, \ldots, q^{N}\right)$, а векторы импульсов - как $p=\left(p_{1}, \ldots, p_{N}\right)$, т. е. $p$ является $N$-мерным векторным пространством над полем действительных чисел $\mathbb{R}$. Для любого целого неотрицательного числа $n$ можно ввести тензорную степень порядка $n$ для $p: p^{\otimes n}=p \otimes p \otimes \cdots \otimes p$; по определению нулевая тензорная степень совпадает с полем: $p^{\otimes 0}=\mathbb{R}$. Прямая сумма всех степеней $p$ является бесконечномерным векторным пространством: $T(p)=\bigoplus_{n=0}^{\infty} p^{\otimes n}$.

Для каждого подпространства $n$-й тензорной степени $p^{\otimes n}$ дуальным пространством является множество тензоров ранга $n$ над $p$, которое можно обозначить как $T^{n}\left(p^{*}\right)$. Прямая сумма всех таких пространств

$$
T\left(p^{*}\right)=\bigoplus_{n=0}^{\infty} T^{n}\left(p^{*}\right)
$$

образует тензорную алгебру, в которой операцию умножения определяют при помощи внешнего произведения тензоров, задающего отображение: $T^{n}\left(p^{*}\right) \otimes T^{k}\left(p^{*}\right) \rightarrow$ $T^{n+k}\left(p^{*}\right)$. В этом пространстве аналогом тензорной степени $n$ является ранг тензоpa. В частности, запись $a_{\mathrm{n}}$ далее будет обозначать тензор $a$ ранга $n$, если нижний индекс напечатан прямым шрифтом. Курсивный шрифт индекса $\left(a^{n}\right)$ обозначает обычную $n$-ю компоненту $a$, т. е. скалярную функцию. Далее также все компоненты всех тензоров полагаются функциями, зависящими от $q$.

Теперь ограничимся подпространством $T\left(p^{*}\right)$, содержащим исключительно симметричные тензоры, - фактор-алгеброй $S\left(p^{*}\right)$. Для двух симметричных тензоров $a_{\mathrm{n}} \in S^{n}\left(p^{*}\right)$ и $b_{\mathrm{k}} \in S^{k}\left(p^{*}\right)$ в качестве операции умножения будем рассматривать симметризованное тензорное произведение

$$
a_{\mathrm{n}} \odot b_{\mathrm{k}}=b_{\mathrm{k}} \odot a_{\mathrm{n}}=\operatorname{Sym}\left(a_{\mathrm{n}} \otimes b_{\mathrm{k}}\right) \in S^{n+k}\left(p^{*}\right),
$$

где $\operatorname{Sym}(\cdot)$ обозначает симметризацию тензора по всем индексам. Тогда $S\left(p^{*}\right)$ будет представлением для симметрической алгебры (см. с. 271-273 в книге [29]). Для нас важно, что $S\left(p^{*}\right)$ также является представлением для полиномов по импульсам без выбора координатного базиса в пространстве $p$. Например, произвольный полином порядка $d$ по импульсам, с коэффициентами, зависящими от координат, можно записать как

$$
I_{d}=\sum_{n=0}^{d} b_{\mathrm{n}}(q) p^{\odot n},
$$

и ему будет соответствовать элемент бесконечномерного векторного пространства $S\left(p^{*}\right)$, у которого только первые $(d+1)$ компонент ненулевые: $I_{d}=\left(b_{0}, \ldots, b_{\mathrm{d}}, 0, \ldots\right)$. В дальнейшем мы будем использовать обе формы записи - в виде полинома и в виде вектора, подразумевая один и тот же элемент из $S\left(p^{*}\right)$.

Именно такой формализм удобен при работе с динамическими системами в теории гравитации, для которых гамильтонианы, как правило, являются полиномами по импульсам, но их конкретный вид зависит от выбора калибровки ${ }^{1)}$ (изменение

1) Наглядный пример получения двух разных на вид динамических систем в зависимости от выбора калибровки в модели можно найти в работе [17]. 
калибровки приводит к другому базису в пространстве $p$ ). Очевидно, что существование первых интегралов не зависит от выбора калибровки, поэтому алгоритм их поиска удобно записать в формализме, не требующем фиксации базиса.

Поскольку нашей задачей является поиск интегралов движения, нужно записать действие скобки Пуассона на $S\left(p^{*}\right)$. Аналогом скобки Ли здесь будет скобка Схоутена для симметричных тензоров [30]. Она отображает два симметричных тензора с рангами $k$ и $n$ в симметричный тензор ранга $n+k-1$ и в координатном представлении имеет следующий вид:

$$
\mathcal{L}_{b_{\mathrm{n}}} a_{\mathrm{k}} \equiv \operatorname{Sym}\left(\sum_{r=1}^{n} \sum_{m_{r}=1}^{N} b^{m_{1} \ldots m_{r} \ldots m_{n}} \partial_{m_{r}} a^{l_{1} \ldots l_{k}}-\sum_{r=1}^{k} \sum_{l_{r}=1}^{N} \partial_{l_{r}} b^{m_{1} \ldots m_{n}} a^{l_{1} \ldots l_{r} \ldots l_{k}}\right) .
$$

Для тензора первого ранга $b_{1}$, т. е. обычного вектора, скобка Схоутена $\mathcal{L}_{b_{1}}(\cdot)$ совпадает с действием производной Ли на симметричные тензоры $a_{\mathrm{k}}$ вдоль вектора $b_{1}$. По построению она является антикоммутативной: $\mathcal{L}_{b_{\mathrm{n}}} a_{\mathrm{k}}=-\mathcal{L}_{a_{\mathrm{k}}} b_{\mathrm{n}}$.

Теперь, вычисляя скобку Пуассона для полиномов стандартным образом, мы можем записать ее действие на $S\left(p^{*}\right)$ при помощи скобки Схоутена. Она будет отображать два элемента $H_{g}=\left(a_{1}, \ldots, a_{\mathrm{g}}, 0, \ldots\right)$ и $I_{d}=\left(b_{0}, \ldots, b_{\mathrm{d}}, 0, \ldots\right)$ в элемент $Y_{g+d-1}=\left(c_{1}, \ldots, c_{\mathrm{g}+\mathrm{d}-1}, 0, \ldots\right)$ следующим образом:

$$
\left\{H_{g}, I_{d}\right\}=Y_{g+d-1}, \quad \text { где } \quad c_{\mathrm{n}}=\sum_{\substack{k=0, \ldots, d, m=0, \ldots, g \\ k+m-1=n}} \mathcal{L}_{b_{\mathrm{k}}} j_{\mathrm{m}} .
$$

Заметим, что симметрическая алгебра $S\left(p^{*}\right)$ имеет естественную градуировку: она разделяется на однородные подпространства $S^{n}\left(p^{*}\right)$, и при этом $S^{n}\left(p^{*}\right) \odot S^{k}\left(p^{*}\right) \rightarrow$ $S^{n+k}\left(p^{*}\right)$. Однако алгебра Пуассона на $S\left(p^{*}\right)$ не будет градуированной, поскольку $\left\{S^{n}\left(p^{*}\right), S^{k}\left(p^{*}\right)\right\} \rightarrow S^{n+k-1}\left(p^{*}\right)$.

Зная действие скобки Пуассона, мы можем записать условия существования полиномиальных первых интегралов. Итак, элемент $I_{d} \in S\left(p^{*}\right)$ является первым интегралом для гамильтониана $H_{g} \in S\left(p^{*}\right)$, если $\left\{H_{g}, I_{d}\right\}=Y_{d+g-1}=0$. Алгоритм Уиттекера трактует это уравнение как условия на все коэффициенты элемента $Y_{d+g-1} \in S\left(p^{*}\right): c_{\mathrm{n}}=0, n=0, \ldots, d+g-1$, поскольку импульсы подразумеваются независимыми. Однако для систем со связями импульсы уже не являются независимыми. В частности, в динамических системах, вытекающих из теории гравитации, присутствует связь $H_{g}=0$. Конечно, она означает, что не все коэффициенты элемента $H_{g} \in S\left(p^{*}\right)$ нулевые, а устанавливает связь между импульсами. Значит, эту связь можно учесть и в уравнении $Y_{d+g-1}=0$, не требуя обращения в нуль всех коэффициентов элемента $Y_{d+g-1} \in S\left(p^{*}\right)$.

Таким образом, мы можем сформулировать условие существования полиномиальных первых интегралов для динамических систем в гравитации с гамильтоновой связью $H_{g}=0$. Элемент $I_{d} \in S\left(p^{*}\right)$ является первым интегралом для гамильтониана $H_{g} \in S\left(p^{*}\right)$ со связью $H_{g}=0$, если

$$
\left\{H_{g}, I_{d}\right\}=Y_{d+g-1}=V_{d-1} \odot H_{g} .
$$

Следовательно, уравнение приобретает $d$ дополнительных коэффициентов $v_{\mathrm{n}}, n=$ $0, \ldots, d-1$, относящихся к вектору $V_{d-1}$ из факторпространства $S\left(p^{*}\right) / H_{g}$. Исключая эти коэффициенты из системы, мы уменьшим число уравнений до $g$. Заметим, 
однако, что эти уравнения являются тензорными, а ранг тензоров будет не выше $d+g-1$, так что в координатном представлении число уравнений по-прежнему будет зависеть от числа $d$.

Надо учесть, что $S\left(p^{*}\right)$, в отличие от кольца многочленов, является моноидом и не допускает применения операции $\odot^{-1}$. Поэтому мы не можем найти $V_{d-1}$ при помощи обычного деления многочленов в столбик. Запишем уравнение (4) в компонентах:

$$
c_{\mathrm{n}}=\sum_{m+k=n} v_{\mathrm{m}} \odot a_{\mathrm{k}}, \quad n=0, \ldots, d+g-1 .
$$

Из этой системы можно последовательно выразить все неизвестные $v_{\mathrm{m}}$ при помощи рекуррентной формулы:

$$
v_{\mathrm{m}} a_{0}=c_{\mathrm{m}}-\sum_{k=1}^{m-k} v_{\mathrm{k}} \odot a_{\mathrm{m}-\mathrm{k}} .
$$

В результате останется только $g$ уравнений на $d+1$ коэффициентов $b_{\mathrm{k}}$ анализируемого полинома $I_{d}$, которые входят в $c_{\mathrm{n}}$ согласно формуле $(3)$.

Теперь мы рассмотрим определенный класс гамильтонианов, для которых система уравнений (5) принимает наиболее простой вид. Назовем четными и нечетными векторами в $S\left(p^{*}\right)$ подмножества векторов с компонентами, являющимися тензорами только четного и нечетного рангов соответственно. Нетрудно заметить, что скобка Пуассона для вектора $I_{d}$ определенной четности с четным гамильтонианом $H_{g}$ порождает векторы $Y_{d+g-1}$ и $V_{d-1}$ другой четности. Вследствие этого можно произвольный вектор $I_{d} \in S\left(p^{*}\right)$ разбить на четную и нечетную части: $I_{2 s+\varepsilon}, s=0,1,2, \ldots$, $\varepsilon=0,1$. Условия (5) существования первого интеграла будут независимыми для каждой части. Так что можно отдельно анализировать четные и нечетные полиномы, эффективным образом вдвое сокращая количество уравнений в системе.

Например, гамильтонианы, имеющие только квадратичную кинетическую и скалярную потенциальную части, встречаются в гравитации наиболее часто:

$$
H=a^{i j}(q) p_{i} p_{j}+U(q) .
$$

Им соответствуют векторы из четного подпространства $S\left(p^{*}\right)$ вида $H_{2}=\left(a_{0}, 0, a_{2}\right.$, $0, \ldots)$, где $a_{0}=U, a_{2}=a^{i j}$, и $H_{2}=a_{2} p^{\odot 2}+a_{0}=0$ на уравнениях движения. Скобка Пуассона для такого гамильтониана с произвольным вектором $I_{d}$ из $S\left(p^{*}\right)$ имеет вид

$$
\left\{H_{2}, I_{d}\right\}=\left(c_{0}, \ldots, c_{\mathrm{d}+1}, 0, \ldots\right), \quad \text { где } \quad c_{\mathrm{n}}=\mathcal{L}_{b_{\mathrm{n}+1}} a_{0}+\mathcal{L}_{b_{\mathrm{n}-1}} a_{2} .
$$

Поскольку гамильтониан содержит всего две ненулевые компоненты, нетрудно при помощи формулы (6) исключить все $v_{\mathrm{m}}$ из системы (5). Тогда для элементов $I_{2 s+\varepsilon}$ из четного и нечетного подпространств система (5) сведется к единственному тензорному уравнению:

$$
\left.\left\{H_{2}, I_{2 s+\varepsilon}\right\}\right|_{H_{2}=0}=0, \quad \text { если } \sum_{n=0}^{s+\varepsilon}\left(-a_{0}\right)^{n}\left(a_{2}\right)^{\odot(s+\varepsilon-n)} \odot c_{2 \mathrm{n}+1-\varepsilon}=0 .
$$

Ранг тензорного уравнения (9) равен $2 s+\varepsilon+1$. Поскольку это симметричный тензор, у него может быть $\left(\begin{array}{c}N+2 s+\varepsilon \\ 2 s+\varepsilon+1\end{array}\right)$ независимых компонент. Очевидно, что число 
отдельных уравнений, содержащихся в системе (9), быстро растет с ростом числа степеней свободы $N$ гамильтониана. Возможно, учет свойств симметричных тензоров [31] позволит преобразовать уравнение (9) к виду, более удобному для решения системы уравнений в частных производных. Мы же далее покажем, как это уравнение выглядит в координатном представлении в простейших случаях для линейных и квадратичных интегралов движения для систем с малым числом степеней свободы.

2.1. Линейные и квадратичные интегралы. Как было показано выше, для квадратичного гамильтониана, $H_{2}=0$, возможно отдельное рассмотрение четных и нечетных полиномиальных интегралов по импульсам вида $I_{2 s+\varepsilon}$. Простейшим примером нечетного интеграла является линейное выражение $I_{1}=b^{i}(q) p_{i}$, когда $s=0, \varepsilon=1$. Теперь мы выпишем в явном виде обычные координатные индексы (в конфигурационном пространстве $\left\{q^{i}\right\}$ ), так что $b^{i}(q)$ обозначает обычное векторное поле.

В данном случае скобка Пуассона (8) содержит следующие ненулевые компоненты: $c_{0}=\mathcal{L}_{b_{1}} a_{0}$ и $c_{2}=\mathcal{L}_{b_{1}} a_{2}$. Заметим, что в координатном представлении первое выражение - это производная от потенциала $a_{0}=U$ вдоль векторного поля $b^{i}$, а второе выражение - это производная Ли тензора $a^{i j}$ вдоль $b^{i}$. Полная система (5) записывается как

$$
\partial_{b} U=v U, \quad \mathcal{L}_{b} a^{i j}=v a^{i j} .
$$

Здесь вектор $V_{0}$ из факторизации (4) содержит только одну ненулевую компоненту $v_{0} \equiv v$ - скаляр. Из такой системы совершенно очевидным образом устраняется параметр $v$, что приводит к уравнению

$$
a^{i j} \partial_{b} U-U \mathcal{L}_{b} a^{i j}=0 .
$$

Естественно, что и общее уравнение (9), которое при подстановке $s=0, \varepsilon=1$ принимает вид $a_{2} c_{0}-a_{0} c_{2}=0$, приводит к точно такому же выражению.

Простейший четный полиномиальный интеграл движения возникает в случае $s=1, \varepsilon=0: I_{2}=b^{i j}(q) p_{i} p_{j}+\mathcal{U}(q)$. Здесь $b_{2} \equiv b^{i j}$ - симметричная матрица, не пропорциональная $a^{i j}$ (иначе это будет сам гамильтониан), $b_{0} \equiv \mathcal{U}-$ скалярная функция.

Скобка Пуассона (8) для векторов $H_{2}$ и $I_{2}$ содержит только две ненулевые компоненты:

$$
\begin{aligned}
& c_{1}=\mathcal{L}_{b_{2}} a_{0}+\mathcal{L}_{b_{0}} a_{2}=2 b^{i k} \partial_{k} U-2 a^{i k} \partial_{k} \mathcal{U}, \\
& c_{3}=\mathcal{L}_{b_{2}} a_{2}=2 \operatorname{Sym}\left(b^{i k} \partial_{k} a^{l m}-\partial_{k} b^{i l} a^{k m}\right) .
\end{aligned}
$$

Полная система (5) условий на коэффициенты имеет вид

$$
c_{1}=v_{1} a_{0}, \quad c_{3}=v_{1} \odot a_{2},
$$

откуда легко выразить $v_{1}$ и получить тензорное уравнение (9): $a_{2} \odot c_{1}-a_{0} c_{3}=0$. В координатном представлении это будет система уравнений:

$$
\operatorname{Sym}\left[a^{l m}\left(b^{i k} \partial_{k} U-a^{i k} \partial_{k} \mathcal{U}\right)-U\left(b^{i k} \partial_{k} a^{l m}-\partial_{k} b^{i l} a^{k m}\right)\right]=0 .
$$

Аналогичным образом по такому алгоритму можно исследовать полиномиальные выражения более высоких порядков по импульсам: сначала по формуле (8) вычисляются компоненты скобки Пуассона $c_{\mathrm{n}}$, а затем решается тензорное уравнение (9). 
В разделе 3 мы применим этот алгоритм для исследования моделей дилатонной гравитации, мы найдем линейные и квадратичные интегралы движения.

\section{3. ПОЛИНОМИАЛЬНЫЕ ИНТЕГРАЛЫ В ДИЛАТОННОЙ ГРАВИТАЦИИ}

Очень часто гравитационные модели рассматриваются при сферической или (тривиальной) цилиндрической пространственной симметрии, что позволяет сформулировать модель на языке двумерной дилатонной гравитации [25]-[28]:

$$
L_{\mathrm{DGS}}=\sqrt{-g}\left[\phi R+W(\phi)\left(\nabla_{\mu} \phi\right)^{2}+Z(\phi) \nabla_{\mu} \psi \nabla^{\mu} \psi+X(\phi, \psi)\right], \quad \mu=(\tau, \sigma),
$$

где $\phi$ - поле дилатона, возникающее вследствие размерной редукции метрического тензора. Заметим, что при более сложных размерных редукциях (например, при нетривиальной цилиндрической редукции) может возникнуть большее количество дилатонных полей. Однако здесь мы ограничиваемся наиболее простым случаем и рассматриваем только одно скалярное поле $\psi$, минимальным образом взаимодействующее с двумерной гравитацией.

Потенциалы взаимодействия дилатона и скалярного поля $Z(\phi), X(\phi, \psi)$, а также потенциал дилатона $W(\phi)$ несут информацию об оригинальной модели и зависят от конкретного вида размерной редукции. Например, многомерное массивное векторное поле после редукции к двум измерениям может быть описано одной скалярной модой [25], поскольку тензор поля пропорционален символу Леви-Чивиты. Однако взаимодействие с метрическим дилатоном будет не таким, как у скалярного поля после редукции.

Как известно, в двумерной теории метрический тензор всегда можно выбрать в следующей форме:

$$
d s^{2}=h\left(-d \tau^{2}+d \sigma^{2}\right)
$$

При переходе к одномерной системе можно рассматривать динамику, соответствующую зависимости только от одной координаты, например $\tau$. В зависимости от выбора знаков в лагранжиане эта координата может играть роль как временно́й, так и пространственной (радиальной) переменной, благодаря чему анализ космологических и статических решений осуществляется единообразно. Для этого одномерный лагранжиан может быть записан в достаточно универсальной форме [25], [32], [28]:

$$
L=s\left[h^{-1} \dot{h} \dot{\phi}+W(\phi) \dot{\psi}^{2}+Z(\varphi) \dot{\psi}^{2}\right]-s^{-1} h X(\varphi, \psi),
$$

которая удобна для дальнейшего исследования. Здесь и далее производная по $\tau$ обозначается точкой над переменной.

Остаточная калибровочная инвариантность лагранжиана, соответствующая репараметризации $\tau$, задается лагранжевым множителем $s$. Вариация по $s$ дает гамильтонову связь, характерную для гравитационных моделей:

$$
H=s\left[h^{-1} \dot{h} \dot{\phi}+W(\phi) \dot{\phi}^{2}+Z(\phi) \dot{\psi}^{2}\right]+s^{-1} h X(\phi, \psi)=0 .
$$

После этого можно фиксировать калибровку для $s$. Наиболее часто выбирают $s=1$, что соответствует изотропной калибровке (16). Однако и другие калибровки могут 
быть удобными для приведения динамической системы (17), (18) к наиболее простому виду.

Прежде всего, можно упростить кинетическую часть, объединив дилатон и метрику в одну новую метрическую переменную $F$, так что $\dot{F}=h^{-1} \dot{h}+W(\phi) \dot{\phi}$. У потенциального слагаемого $U(\phi, \psi)$ появится дополнительный множитель

$$
\Omega(\phi)=\exp \left(-\int W(\phi) d \phi\right) .
$$

Затем зависимость кинетической части от дилатона может быть упрощена при помощи выбора новой дилатонной переменной $d \varphi=s(\phi) d \phi$.

В новых переменных динамическая система (17), (18) записывается как

$$
L=\dot{F} \dot{\varphi}-s Z \dot{\psi}^{2}-s^{-1} \Omega X e^{F}, \quad H=\dot{F} \dot{\varphi}-s Z \dot{\psi}^{2}+s^{-1} \Omega X e^{F}=0 .
$$

В фазовом пространстве $q^{i}=(F, \varphi, \psi), p_{i}=(\dot{\varphi}, \dot{F},-2 s Z \dot{\psi})$ гамильтониан соответствует виду $H_{2}=0$, рассмотренному в разделе 2 (см. (7)), в котором $a^{12}=a^{21}=1 / 2$, $a^{33}=-1 / 4 s Z, U=s^{-1} \Omega X e^{F}$.

В безмассовом случае, когда потенциал $X$ не зависит от скалярного поля $\psi$, удобно устранить эту переменную ${ }^{2)}$ при помощи уравнений движения. Для безмассового скалярного поля уравнение Лагранжа тривиально интегрируется:

$$
-2 s Z \dot{\psi}=2 Y_{0}=\text { const }
$$

где $Y_{0}$ - параметр, задающий “заряд” скалярного поля. Можно записать эффективный лагранжиан, где вместо кинетического слагаемого $-s Z \dot{\psi}^{2}$ будет добавочное слагаемое в потенциальной части, так что динамическая система (19) в безмассовом случае будет описываться гамильтонианом

$$
H=p_{F} p_{\varphi}+s^{-1} \Omega X e^{F}-(s Z)^{-1} Y_{0}^{2}=0 .
$$

Теперь мы применим алгоритм, рассмотренный в разделе 2 , к динамической системе (19). С его помощью мы не просто ищем интегралы для конкретной модели, а можем протестировать сразу весь класс моделей вида (19) на наличие интегралов движения в зависимости от потенциалов взаимодействия $\Omega X$ и $Z$. Такой подход позволяет выделить те модели, которые обладают дополнительными симметриями, а значит, являются более удобными для дальнейшего анализа. Мы ограничимся только интегралами, линейными и квадратичными по импульсам, поскольку соответствующие симметрии достаточно распространены и имеют вполне наглядный смысл.

3.1. Линейные интегралы. Условия существования линейных интегралов заданы системой (10). Сначала отдельно рассмотрим безмассовый случай (20). Уравнение $\mathcal{L}_{b} a^{i j}=v a^{i j}$ является общим для всего этого класса моделей дилатонной гравитации: в безмассовом случае в переменных $F, \varphi$ кинетическая матрица $a^{i j}$

${ }^{2)}$ В разделе 2 мы показали, что число уравнений для определения коэффициентов интегралов движения значительно возрастает при росте числа переменных. 
с ненулевыми компонентами $a^{12}=a^{21}=1 / 2$ одинакова для всех моделей. Решение выглядит очень просто:

$$
b^{i}=\left(b_{F}(F), b_{\varphi}(\varphi)\right), \quad v=-\left(b_{F}\right)_{F}^{\prime}-\left(b_{\varphi}\right)_{\varphi}^{\prime},
$$

где $b_{F}, b_{\varphi}$ являются произвольными функциями своих аргументов.

Теперь решим оставшееся уравнение $\partial_{b} U=v U$, где потенциал дилатонной безмассовой гравитации имеет вид

$$
U=s^{-1} \Omega X e^{F}-(s Z)^{-1} Y_{0}^{2} .
$$

За исключением тривиальных случаев, когда одно из потенциальных слагаемых обращается в нуль или они оба равны константе, мы получаем два семейства решений:

$$
\begin{aligned}
& b^{1}=\frac{C_{2} e^{F}-C_{2} C_{3} F+C_{4}}{e^{F}-C_{3}}, \quad b^{2}=\frac{C_{2} \beta-C_{1}}{\beta^{\prime}}, \quad s^{-1} \Omega X=\beta^{\prime}, \quad(s Z)^{-1} Y_{0}^{2}=C_{3} \beta^{\prime} ; \\
& b^{1}=-C_{2}, \quad b^{2}=\frac{C_{2} \beta-C_{1}}{\beta^{\prime}}, \quad s^{-1} \Omega X=\beta^{\prime}, \quad(s Z)^{-1} Y_{0}^{2}=\frac{\beta^{\prime}}{C_{2} \beta-C_{1}} .
\end{aligned}
$$

Эти семейства параметризуются произвольной функцией $\beta(\varphi)$. Благодаря наличию этой степени свободы появляется возможность среди различных моделей искать такую, которая допускает дополнительный интеграл движения. Напомним, что сам интеграл строится при помощи коэффициентов $b^{i}: I_{1}=b^{i} p_{i}$.

Подобным образом можно исследовать и массивную дилатонную гравитацию с потенциалом, зависящим от скалярного поля (19). Для удобства выберем калибровку $s=1 / Z$. Тогда, как и в безмассовом случае, кинетическая матрица $a^{i j}$ с ненулевыми компонентами $a^{12}=a^{21}=1 / 2, a^{33}=-1 / 4$ будет общей для всех массивных моделей. Решением уравнения $\mathcal{L}_{b} a^{i j}=v a^{i j}$ будет семейство

$$
\begin{aligned}
v & =-C_{1} F-C_{6} \varphi-2 C_{4} \psi-C_{5}-C_{9}, \\
b_{F} & =\frac{C_{6} \psi^{2}}{2}+\left(C_{4} F+C_{7}\right) \psi+\frac{C_{1} F^{2}}{2}+C_{9} F+C_{8}, \\
b_{\varphi} & =\frac{C_{1} \psi^{2}}{2}+\left(C_{2}+C_{4} \varphi\right) \psi+\frac{C_{6} \varphi^{2}}{2}+C_{5} \varphi+C_{3}, \\
b_{\psi} & =\frac{C_{4} \psi^{2}}{2}+\frac{\left(C_{1} F+C_{6} \varphi+C_{5}+C_{9}\right) \psi}{2}+\frac{\left(C_{4} \varphi+C_{2}\right) F}{2}+\frac{C_{7} \varphi}{2}+C_{10},
\end{aligned}
$$

параметризуемое десятью константами $C_{k}$ (если не фиксировать калибровку, то решение также будет параметризовано некоторой произвольной функцией). Это семейство решений содержит все возможные линейные интегралы в массивной дилатонной гравитации (19).

Для определения потенциалов, при которых реализуются те или иные интегралы, необходимо еще учесть уравнение $\partial_{b} U=v U$, где $U=Z \Omega X e^{F}$ в выбранной калибровке. Рассмотрение этого десятипараметрического семейства выходит за рамки нашего исследования, хотя и не представляет никаких технических трудностей. Комбинируя параметры, можно подобрать такие уравнения, решениями которых будут популярные потенциалы степенного или экспоненциального вида, а также более экзотические. 
Полученный результат можно использовать и другим образом. Семейство (23) определяет все допустимые симметрии в рассматриваемой массивной дилатонной гравитации, соответствующие циклическим переменным. Если из каких-либо физических соображений требуется построить модель с определенной симметрией подобного типа, то нужно посмотреть, при каких значениях параметров $C_{i}$ ее можно реализовать, а затем решить уравнение $\partial_{b} U=v U$ относительно потенциала, получив таким образом информацию для дальнейшего построения модели. Однако может выясниться, что для реализации этой симметрии в принципе не подходит класс моделей вида (17). Например, окажется, что в модель необходимо добавить еще одно скалярное поле.

3.2. Квадратичные интегралы. Полиномы второго порядка $I_{2}=b^{i k} p_{i} p_{k}+\mathcal{U}$ в безмассовом случае, когда присутствуют только две динамические переменные, параметризуются четырьмя функциями: $b^{11}, b^{12}, b^{22}$ и $V$. Очевидно, что сам гамильтониан также является квадратичным интегралом, поэтому имеет смысл рассматривать только интегралы с равной нулю компонентой $b^{12}$. В отличие от линейного случая, где мы решали систему (10) по шагам, мы сразу перейдем к решению итогового уравнения (14), откуда исключены параметры $v^{i}$.

Заметим, что квадратичный интеграл также может быть квадратом линейного интеграла, что не несет новой информации о системе. Поэтому нас интересует случай, когда квадратичный интеграл не сводится ни к гамильтониану, ни к квадрату линейного интеграла. Подставив $a^{12}=a^{21}=1 / 2, U=s^{-1} \Omega X e^{F}-(s Z)^{-1} Y_{0}^{2}$ в уравнение (14), мы обнаружили, что такие новые квадратичные интегралы соответствуют случаю, когда $b^{22}$ не обращается в нуль. Например, подмножество интегралов, у которых в матрице $b^{i j}$ только компонента $b^{22}$ не равна нулю, выглядит следующим образом:

$$
\begin{aligned}
b^{22} & =\left[\beta^{\prime}(\varphi)\right]^{-2}, & \mathcal{U} & =2 C_{1} F+2 C_{2} e^{F}, \\
(s Z)^{-1} Y_{0}^{2} & =\left(C_{3}-C_{1} \beta\right) \beta^{\prime}, & s^{-1} \Omega X & =\left(C_{4}+C_{2} \beta\right) \beta^{\prime} .
\end{aligned}
$$

Это семейство параметризуется функцией $\beta(\varphi)$ и четырьмя константами $C_{k}$. Соответствующие квадратичные интегралы (впервые найденные в работе [26]) имеют вид

$$
I_{2}=\left(\frac{p_{2}}{\beta^{\prime}}\right)^{2}+2 C_{1} F+2 C_{2} e^{F}, \quad\left\{H, I_{2}\right\}=\frac{2 H p_{2} \beta^{\prime \prime}}{\beta^{\prime 3}}
$$

Мы видим, что эти интегралы сохраняются именно вследствие того, что их скобка Пуассона с гамильтонианом допускает факторизацию (4) и обращается в нуль, поскольку гамильтониан обращается в нуль.

В случае массивных моделей дилатонной гравитации (19), где присутствуют три динамические переменные, квадратичные интегралы параметризуются уже семью функциями $b^{i k}$ и $\mathcal{U}$. Поиск общего решения выходит за пределы нашего исследования, поэтому мы приведем только несколько простых примеров.

Сначала найдем аналог интеграла (25) в массивной модели. Уравнение (14) при единственной ненулевой квадратичной компоненте $b^{22}$ имеет решение

$$
\begin{gathered}
b^{22}=\left[\beta^{\prime}(\varphi)\right]^{-2}, \quad \mathcal{U}=2 C_{2} e^{F}, \\
(s Z)^{-1}=C_{3} \beta^{\prime}, \quad s^{-1} \Omega X=\left(f(\psi)+C_{2} \beta\right) \beta^{\prime},
\end{gathered}
$$


определенное с точностью до трех констант $C_{k}$ и произвольной функции $\beta(\varphi)$. Как мы видим, потенциал скалярного поля может иметь определенную зависимость от $\psi$ с точностью до произвольной функции $f$.

Теперь рассмотрим, например, вариант, когда единственной ненулевой квадратичной компонентой является $b^{23}$. Тогда решением уравнения (14) будет

$$
\begin{aligned}
b^{23} & =\left[\beta^{\prime}(\varphi)\right]^{-1}, & \mathcal{U} & =\left(4 C_{1} \psi+2 C_{2}\right) e^{F}, \\
(s Z)^{-1} & =C_{3} \beta^{\prime}, & s^{-1} \Omega X & =\left(C_{1} \psi^{2}+C_{2} \psi+C_{4}-C_{1} C_{3} \beta\right) \beta^{\prime} .
\end{aligned}
$$

Как и прежде, $C_{k}$ - константы, а $\beta(\varphi)$ - произвольная функция.

Сравнивая два примера (26), (27) для массивной модели, нетрудно заметить, что решение для потенциала $s Z$ в обоих случаях имеет одинаковый вид, а решение для потенциала $s^{-1} \Omega X$ будет одинаковым при выборе $f(\psi)$ в виде квадратичного полинома. Таким образом, мы обнаружили модель дилатонной гравитации, которая допускает сразу два квадратичных интеграла движения:

$$
\begin{aligned}
& H=p_{1} p_{2}-\frac{C_{3} \beta^{\prime}(\varphi) p_{3}^{2}}{4}+\left[C_{1} \psi^{2}+C_{2} \psi+C_{4}-C_{1} C_{3} \beta(\varphi)\right] \beta^{\prime}(\varphi) e^{F}=0, \\
& I_{2}^{(1)}=\frac{p_{2}^{2}}{\beta^{\prime 2}}-2 C_{1} C_{3} e^{F}, \quad I_{2}^{(2)}=\frac{2 p_{2} p_{3}}{\beta^{\prime}}+\left(4 C_{1} \psi+2 C_{2}\right) e^{F}, \\
& \left\{H, I_{2}^{(1)}\right\}=\frac{2 H p_{2} \beta^{\prime \prime}}{\beta^{\prime 3}}, \quad\left\{H, I_{2}^{(2)}\right\}=\frac{2 H p_{3} \beta^{\prime \prime}}{\beta^{\prime 2}}, \quad\left\{I_{2}^{(1)}, I_{2}^{(2)}\right\}=0 .
\end{aligned}
$$

И в этом случае оба интеграла сохраняются вследствие того, что результат действия скобки Пуассона содержит в качестве множителя гамильтониан, который обращается в нуль. Оказывается, что они также находятся в инволюции, благодаря чему система с гамильтонианом (28) является интегрируемой. При вполне реалистичном степенном потенциале дальнейшее исследование этой модели может представлять интерес.

\section{4. ЗАКЛЮЧЕНИЕ}

В работе был исследован вопрос о существовании первых интегралов для достаточно широкого класса дилатонных моделей гравитации с одним скалярным полем. Для него мы нашли полное десятипараметрическое семейство линейных по импульсу первых интегралов. Такой результат, например, дает представление о возможности реализации соответствующих симметрий в изучаемых моделях дилатонной гравитации. Также для этого класса моделей были представлены случаи возникновения квадратичных по импульсу первых интегралов, что позволило найти пример нетривиальной интегрируемой модели с достаточно реалистичным степенным потенциалом взаимодействия дилатона и скалярного поля.

Особенностью выбранного нами подхода является алгоритм перебора возможных интегралов движения, легко реализуемый на компьютере при помощи современных пакетов символьных вычислений. Это позволяет находить интегралы движения в случае достаточно специфических, неочевидных симметрий.

В разделе 1 мы отмечали, что существующие в настоящее время тенденции в области построения гравитационных моделей заключаются в определенном усложнении структуры моделей и рассмотрении набора дополнительных полей. Отметим, что 
наш подход позволяет, при сохранении такого существенного свойства, как полиномиальность по импульсам гамильтониана динамической системы, изучать и более сложные модели. Число степеней свободы, зависящее от рассматриваемого набора полей материи, и структура гамильтониана являются просто входными данными для предложенной алгоритмической процедуры (6), (8).

Знание первых интегралов дает важную информацию о системе: сигнализирует о внутренних симметриях и облегчает анализ пространства классических решений, что особенно важно, поскольку большинство моделей являются неинтегрируемыми. С помощью первых интегралов можно выделять модели, обладающие различными полезными свойствами, например, демонстрирующие поведение типа аттрактора [33], обладающие устойчивостью и не требующие тонкой настройки параметров модели для реализации нужной динамики.

Благодаря этому можно установить связь между классами моделей в теориях дилатонной гравитации и свойствами пространств их классических решений. Это значительно упростит процедуру выбора моделей для фитирования наблюдательных данных и их дальнейшее рассмотрение на более фундаментальном уровне.

Благодарности. Автор выражает глубокую благодарность проф. А. Т. Филиппову за полезное обсуждение и ценные замечания.

\section{Список литературы}

[1] P. A. R. Ade, R. W. Aikin, M. Amiri et al. [BICEP2 Collab.], BICEP2 II: Experiment and three-year data set, arXiv: 1403.4302 .

[2] T. Delubac, J. E. Bautista, N. G. Busca et al., Baryon acoustic oscillations in the Lya forest of BOSS DR11 quasars, arXiv: 1404.1801.

[3] D. K. Hazra, A. Shafieloo, G. F. Smoot, A. A. Starobinsky, JCAP, 08 (2014), 048, arXiv: 1405.2012.

[4] K. Bamba, S. 'i. Nojiri, S. D. Odintsov, Phys. Lett. B, 737 (2014), 374-378, arXiv: 1406.2417.

[5] R. Kallosh, A. Linde, A. Westphal, Phys. Rev. D, 90:2 (2014), 023534, 12 pp., arXiv: 1405.0270.

[6] V. Sahni, A. Shafieloo, A. A. Starobinsky, Astrophys. J., 793:2 (2014), L40, arXiv: 1406.2209.

[7] А. Д. Линде, Физика элементарных частии, и инфляиионная космология, Наука, М., 1990; A. Linde, "Particle physics and inflationary cosmology", Proceedings of the Fourth Seminar on Quantum Gravity (Moscow, May 25-29, 1987), Contemp. Concepts Phys., 5, eds. M. A. Markov, V. A. Berezin, V. P. Frolov, Word Sci., Singapore, 2005, 736-746, arXiv: hep-th/0503203.

[8] V. Sahni, "Dark matter and dark energy", The Physics of the Early Universe, Lecture Notes in Physics, 653, ed. E. Papantonopoulos, Springer, Berlin, 2004, 141-179, arXiv: astro-ph/0403324.

[9] D. V. Gal'tsov, E. A. Davydov, Internat. J. Modern Phys. Conf. Ser., 14 (2012), 316-325, arXiv: 1112.2943.

[10] A. Maleknejad, M. M. Sheikh-Jabbari, Phys. Lett. B, 723:1-3 (2013), 224-228, arXiv: 1102.1513.

[11] S. Nojiri, S. D. Odintsov, Internat. J. Geom. Meth. Modern Phys., 4:1 (2007), 115-145, arXiv: hep-th/0601213.

[12] S. F. Hassan, R. A. Rosen, JHEP, 07 (2011), 009, 22 pp., arXiv: 1103.6055.

[13] P. Horava, Phys. Rev. D, 79:8 (2009), 084008, 15 pp., arXiv: 0901.3775.

[14] А. Т. Филиппов, ТМФ, 163:3 (2010), 430-448, arXiv: 1003.0782. 
[15] J. A. Harvey, A. Strominger, "Quantum aspects of black holes", String Theory and Quantum Gravity'92 (Trieste, Italy, 30 March-10 April, 1992), eds. J. Harvey, R. Lengo, K. S. Narain, S. Randjbar-Daemi, H. Verlinde, World Sci., Singapore, 1993, 122-?, arXiv: hep-th/9209055.

[16] D. Grumiller, W. Kummer, D. V. Vassilevich, Phys. Rep., 369:4 (2002), 327-430, arXiv: hep-th/0204253.

[17] R. Kallosh, A. Linde, JCAP, 01 (2014), 020, 18 pp., arXiv: 1311.3326.

[18] Э. Уиттекер, Аналитическал динамика, Эдиториал УРСС, М., 2004.

[19] M. Hénon, C. Heiles, Astronom. J., 69 (1964), 73-79.

[20] M. Toda, J. Phys. Soc. Japan, 22:2 (1967), 431-436.

[21] S. C. Mishra, F. Chand, Pramana, 66:3 (2006), 601-607.

[22] A. V. Tsiganov, J. Phys. A: Math. Gen., 33:41 (2000), 7407-7422, arXiv: nlin/0001053.

[23] C. R. Holt, J. Math. Phys., 23:6 (1982), 1037-1046.

[24] В. В. Козлов, Д. В. Трещёв, Матем. сб., 135(177):1 (1988), 119-138.

[25] А. Т. Филиппов, ТМФ, 177:2 (2013), 323-352, arXiv: 1302.6372.

[26] A. T. Filippov, Modern Phys. Lett. A, 11:21 (1996), 1691-1704, arXiv: hep-th/9605008; Internat. J. Modern Phys. A, 12:1 (1997), 13-22, arXiv: gr-qc/9612058.

[27] В. Де Альфаро, А. Т. Филиппов, ТМФ, 153:3 (2007), 422-452, arXiv: hep-th/0612258.

[28] A. T. Filippov, Integrals of equations for cosmological and static reductions in generalized theories of gravity, arXiv: 1403.6815 .

[29] А.И. Кострикин, Ю.И. Манин, Линейная алгебра и геометрия, Наука, М., 1986.

[30] M. Dubois-Violette, P.W. Michor, Indag. Math. (N.S.), 6:1 (1995), 51-66, arXiv: alg-geom/9401006.

[31] P. Comon, G. Golub, L. H. Lim, B. Mourrain, SIAM J. Matrix Anal. Appl., 30:3 (2008), 1254-1279, arXiv: 0802.1681.

[32] E. A. Davydov, A. T. Filippov, Gravit. Cosmol., 19:4 (2013), 209-218, arXiv: 1302.6969.

[33] R. Kallosh, A. Linde, D. Roest, Phys. Rev. Lett., 112:1 (2014), 011303, arXiv: 1310.3950. 